\title{
A study protocol for HEalth-Related quality of life-intervention in survivors of Breast and other cancers experiencing cancer- related fatigue using TraditionAL Chinese Medicine: the HERBAL trial
}

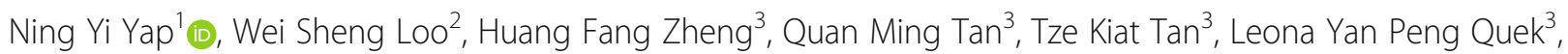

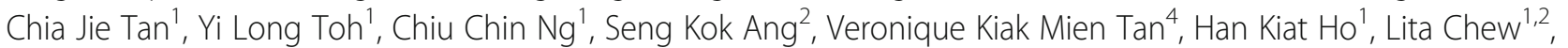
Kiley Wei-Jen Loh ${ }^{5}$, Tira Jing Ying $\operatorname{Tan}^{5}$ and Alexandre Chan ${ }^{2,6^{*}}$

\begin{abstract}
Background: Cancer-related fatigue (CRF) is a debilitating condition which commonly affects cancer survivors. The management of CRF remains a challenge due to the lack of effective pharmacological interventions. Traditional Chinese medicine (TCM) could be a potential therapeutic option for CRF. The modified Xiang Bei Yang Rong Tang (XBYRT) is a TCM herbal decoction, formulated to improve fatigue symptoms in cancer survivors. This clinical trial aims to evaluate the efficacy and safety of XBYRT in improving CRF and quality of life (QOL) of cancer survivors.

Methods: This is a single centre, randomized, double-blind, placebo-controlled, parallel trial. Eighty cancer survivors will be recruited and randomized to receive the XBYRT or placebo decoction, in a ratio of 1:1. Participants will consume the XBYRT/placebo decoction daily for 8 weeks and undergo assessments at baseline and 4, 8 and 10 weeks after baseline. The participants will be assessed for patient-reported outcomes (PRO), blood biomarkers and adverse events at each time point. The primary outcome is the overall health and QOL status, at 8 weeks follow-up. The secondary outcomes are the effects of XBYRT on fatigue levels, cancer-related cognitive impairment and QOL, as assessed by PRO. The incidence of adverse events and the effects of the XBYRT decoction on blood biomarkers associated with CRF will also be evaluated.

Discussion: Efficacy and safety outcomes from this trial will provide important clinical data to guide future large-scale randomized controlled trials, and the evaluation of the objective blood biomarkers can help to delineate the biological mechanisms of CRF.
\end{abstract}

Trial registration number: ClinicalTrials.gov NCT04104113. Registered on 26 September 2019

Keywords: Randomized controlled trial, Cancer survivors, Quality of life, Cancer-related fatigue, Traditional Chinese medicine

\footnotetext{
* Correspondence: a.chan@uci.edu

${ }^{2}$ Department of Pharmacy, National Cancer Centre Singapore, Singapore, Singapore

${ }^{6}$ Department of Clinical Pharmacy Practice, University of California, Irvine, USA

Full list of author information is available at the end of the article
}

(c) The Author(s). 2020 Open Access This article is licensed under a Creative Commons Attribution 4.0 International License, which permits use, sharing, adaptation, distribution and reproduction in any medium or format, as long as you give appropriate credit to the original author(s) and the source, provide a link to the Creative Commons licence, and indicate if changes were made. The images or other third party material in this article are included in the article's Creative Commons licence, unless indicated otherwise in a credit line to the material. If material is not included in the article's Creative Commons licence and your intended use is not permitted by statutory regulation or exceeds the permitted use, you will need to obtain permission directly from the copyright holder. To view a copy of this licence, visit http://creativecommons.org/licenses/by/4.0/. The Creative Commons Public Domain Dedication waiver (http://creativecommons.org/publicdomain/zero/1.0/) applies to the data made available in this article, unless otherwise stated in a credit line to the data. 


\section{Introduction}

Advances in diagnosis and treatment have resulted in better survival rates and an increase in the number of cancer survivors [1]. Cancer survivors often experience a wide range of radiotherapy or chemotherapy-induced side effects which may persist for years even after the cessation of treatment. Cancer-related fatigue (CRF) has been reported as the most distressing symptom experienced by cancer patients and can have debilitating physical, emotional and cognitive effects which negatively impact the patients' quality of life (QOL) $[2,3]$. Up to $45 \%$ of cancer patients suffer from clinically significant CRF during treatment, and approximately 29\% still experience fatigue after the completion of treatment [4].

The pathophysiology and mechanisms leading to CRF and the factors causing persistent fatigue in cancer survivors are not well understood, despite it being a highly prevalent symptom in cancer patients and survivors. This is because the pathophysiology underlying CRF is complex involving psychological, physical and biological factors. One of the postulated biological factors contributing to the development of CRF is inflammation from immune activation caused by cancer and its treatment. Dysregulation of circulating inflammatory markers and cytokines such as interleukin-1 receptor antagonist (IL-1RA), interleukin-6 (IL-6) and C-reactive protein (CRP) has been reported in cancer patients and survivors experiencing CRF [5-8]. Chemotherapy, along with a pro-inflammatory environment, induces a number of downstream toxic effects, including an increase in reactive oxygen species which can result in mitochondrial impairment [9-11]. Mitochondrial dysfunction has been implicated as one of the possible biological factors of chronic fatigue syndrome and CRF [12-14].

In Asia, many cancer patients seek complementary and alternative medicine (CAM) to manage their cancerrelated symptoms or improve their general health, and one of the most commonly used CAM is traditional Chinese medicine (TCM) [15-17]. According to the TCM concept, CRF is characterized by the deficiency of qi, the vital energy of the body, along with blood deficiency [18]. Chemotherapy and radiotherapy deplete both qi and blood by indiscriminately destroying growing cells, damaging the bone marrow and weakening the body. Qi deficiency was found to be associated with CRF and a poorer QOL in cancer patients [18, 19]. In addition, a few studies looking at various TCM decoctions have shown effectiveness in improving fatigue levels in cancer patients [20-23]. However, the efficacy of TCM is not backed with robust clinical evidence and safety data as most of these studies suffer from the lack of adequate control and blinding which might introduce bias in the results. Therefore, more well-designed randomized controlled clinical trials are needed to evaluate the efficacy and safety of TCM in treating CRF.
The TCM formula for this randomized controlled trial is a modification of Xiang Bei Yang Rong Tang (香贝养荣 汤). Xiang Bei Yang Rong Tang (XBYRT) was described in the ancient TCM text "Yi Zong Jin Jian" volume 64 and is used for nourishing qi and blood [24]. The 15 herbal components in the modified XBYRT formula were selected based on their ability to augment qi, nourish the blood, improve appetite and calm the mind which can help to alleviate CRF symptoms in cancer survivors.

The primary objective of this trial is to evaluate the efficacy of XBYRT in improving the overall health status and QOL in a cohort of cancer survivors, assessed using the global health status of the European Organization for Research and Treatment of Cancer Quality of Life Questionnaire (EORTC QLQ-C30), at 8 weeks follow-up. The secondary objectives of this trial are to determine the effects of XBYRT on fatigue levels, cancer-related cognitive impairment and QOL. In addition, the safety outcomes associated with XBYRT and the impact of the TCM decoction on biomarkers associated with CRF will be evaluated.

\section{Methods \\ Study design}

This is a single-centre randomized, double-blind, placebocontrolled, parallel trial, and the protocol for this trial has been prepared in accordance with the Standard Protocol Items: Recommendations for Interventional Trials (SPIR IT)-TCM extension statement 2018 (Additional file 1) [25]. This study protocol has received ethical approval from the Centralised Institutional Review Board (2019/2135) and is registered in ClinicalTrials.gov (NCT04104113). Written informed consent will be obtained from all study participants. A total of 80 cancer survivors will be enrolled from the outpatient oncology clinics at the National Cancer Centre Singapore (NCCS), and the recruitment and followup period for this trial will be from October 2019 to December 2021.

\section{Participants}

The target study participants are cancer survivors who complain of fatigue and will be recruited through referrals from oncologists. Cancer survivors will be screened for fatigue using a single item, where patients will be asked to rate their fatigue on a scale of 0 to 10 over the past 7 days. A score of 0 means no fatigue, and mild fatigue is indicated as a score of 1 to 3 , moderate fatigue as 4 to 6 and severe fatigue as 7 to 10 [26]. Patients with ratings $\geq 4$ are considered as experiencing significant fatigue. Potential participants will also be screened by certified TCM physicians based on TCM syndrome differentiation. The eligibility criteria are as follows:

1. Age $\geq 21$ years

2. Clinically diagnosed cancer (stages I-III) 
3. Completed surgery/chemotherapy/radiotherapy for at least 1 month

4. At least 1 month after starting on aromatase inhibitors or ovarian suppression for breast cancer survivors

5. Not expected to receive surgery/chemotherapy/ radiotherapy for the next 10 weeks

6. Fatigue screening score $\geq 4$ for the past 7 days

7. Life expectancy $\geq 3$ months

8. Patients who satisfy TCM syndrome differentiation as qi and blood deficiency: experience 2 major symptoms coupled with typical tongue and pulse conditions; 2 major symptoms and 1 possible symptom coupled with tongue and pulse conditions; and 1 major symptom and at least 2 possible symptoms coupled with tongue and pulse conditions

9. Able to read and understand English or Mandarin

The exclusion criteria are as follows:

1. Cancer recurrence and/or metastasis.

2. Untreated co-morbidities causing fatigue (e.g. severe anaemia, thyroid disorder).

3. On medications that cause fatigue (e.g. beta blockers).

4. Patients on warfarin.

5. Cancer survivors receiving adjuvant therapy during the study period. Aromatase inhibitors and antihuman epidermal growth factor receptor 2 (HER2) monoclonal antibodies are acceptable.

6. Receiving or planning to receive treatment from other TCM practitioners during the study period.

7. Breastfeeding or intending to conceive/get pregnant during the study treatment period.

8. Patients who present with yin deficiency and excess syndromes (e.g. phlegm-dampness, blood stasis, toxic heat and qi stagnation).

\section{Intervention}

Study participants will take either the assigned XBYRT or placebo which is prepared as granules once daily for a duration of 8 weeks. Participants are required to dissolve the XBYRT or placebo granules in a cup of hot water and consume the decoction. During the study period, participants will be asked to refrain from taking other supplements or herbal medicines.

The experimental group will be given the XBYRT granules. The formulation, the TCM indication and the daily dosage of the XBYRT are displayed in Table 1. The study dosage is selected based on the safety and efficacy guidelines from the Pharmacopoeia of the People's Republic of China and General Eleventh Five-Year National Planning Textbook for Higher Education: Chinese Materia Medica $[27,28]$. The dosage indicated in Table 1 represents the amount of raw materials required for the extraction of the granules. The dried granules are extracted from the boiled aqueous concentrate of the raw materials. The final daily dosage consists of $24 \mathrm{~g}$ of extracted granules.

The control group will receive a daily dosage containing $24 \mathrm{~g}$ of placebo granules. The placebo granules comprise of $5 \%$ of the herbal components, $95 \%$ maltodextrin, colourant and $0.002 \%$ denatonium benzoate as bitterant. This is to ensure that the placebo possesses the taste and smell of the herbal components. For this reason, it is a common practice in TCM trials to incorporate herbal components ranging from 5 to $10 \%$ in the placebo decoction, and this concentration is unlikely to exert a therapeutic effect $[29,30]$. The placebo will be colour adjusted to match the XBYRT granules, and the packaging for the XBYRT and placebo granules will be similar in appearance. In order to encourage adherence to the study intervention, participants will be reminded to take their daily dosages and record their missed dosages.

Both the XBYRT and placebo granules will be manufactured by Kinhong Pte Ltd., Singapore, a Good Manufacturing Practices-certified manufacturer. A certificate of analysis containing the results on component identification, moisture content and the absence of heavy metal and microbial contamination will be available for each batch of XBYRT/placebo granules manufactured.

\section{Randomization, allocation concealment and blinding}

Recruited participants will be randomized into the XBYRT or placebo treatment arm by block randomization at a 1:1 ratio, with a block size of 10 . The consultant physicians, study team, trial pharmacists and study participants are blinded to the intervention to minimize potential bias. Randomization and blinding will be performed by a third party clinical trial service provider, Singapore Clinical Research Institute, using the sealed envelope method. Participants will collect the granules from the clinical trial pharmacy at NCCS based on their pre-assigned numbers.

\section{Sample size}

In order to ensure that the sample size is adequately powered to estimate a reliable standard deviation (SD) for a phase III trial, Teare et al. has recommended to include at least 70 subjects ( 35 per arm) for estimating the SD for a continuous outcome [31]. Therefore, to account for a $10 \%$ dropout, a total sample size of 80 patients (rounded up from 78), with 40 participants on each arm, is required for this study.

\section{Assessments}

Participants will be assessed at four time points: baseline and 4 weeks, 8 weeks and 10 weeks after baseline (Fig. 1). At the baseline visit, the participant's demographics, medical history, cancer diagnosis and concomitant medications will be recorded in a standardized data collection form. The participants will be assessed for patient-reported outcomes 
Table 1 Formula and components of the modified Xiang Bei Yang Rong Tang decoction

\begin{tabular}{|c|c|c|c|c|}
\hline $\begin{array}{l}\text { Chinese } \\
\text { name }\end{array}$ & $\begin{array}{l}\text { Chinese name } \\
\text { (Pinyin) }\end{array}$ & Scientific name & $\begin{array}{l}\text { Dosage } \\
\text { (g) }\end{array}$ & Purported effect \\
\hline 黄芪 & Huang Qi & Radix Astragaliseu Hedysari & 15 & $\begin{array}{l}\text { Augments qi and raises yang, augments defensive qi, consolidates the } \\
\text { superficies, promotes drainage of pus and healing, facilitates water } \\
\text { movement and reduces swelling }\end{array}$ \\
\hline 党参 & Dang Shen & Radix Codonopsis Pilosulae & 15 & Tonifies the middle jiao, augments qi and generates fluids and blood \\
\hline 白术 & Bai zhu & $\begin{array}{l}\text { Rhizoma Atractylodis } \\
\text { Macrocephalae }\end{array}$ & 12 & $\begin{array}{l}\text { Augments qi, strengthens the spleen, dries dampness, promotes } \\
\text { diuresis and stops sweating }\end{array}$ \\
\hline 获苓 & Fu Ling & Poria & 15 & $\begin{array}{l}\text { Drains water, dissipates dampness, strengthens the spleen and calms } \\
\text { the mind }\end{array}$ \\
\hline 白药 & Bai Shao & Radix Paeoniae Alba & 15 & $\begin{array}{l}\text { Nourishes the blood, retains yin, soothes the liver and relieves pain, } \\
\text { and stops excessive perspiration }\end{array}$ \\
\hline 枸杞子 & Gou Qi zi & Fructus Lycii & 12 & Nourishes the liver and kidney, clears the eyes and moistens the lung \\
\hline 女贞子 & Nü Zhen Zi & Fructus Ligustri Lucidi & 12 & Tonifies the liver and kidney, cools heat and clears the eye \\
\hline 车前子 & Che Qian Zi & Plantago asiatica & 12 & Induces diuresis, drain dampness, improve vision and resolve phlegm \\
\hline 鸡内金 & Ji Nei Jin & $\begin{array}{l}\text { Endothelium Corneum Gigeriae } \\
\text { Galli }\end{array}$ & 10 & $\begin{array}{l}\text { Promotes digestion and invigorate spleen, arrest seminal emission } \\
\text { and relieve enuresis }\end{array}$ \\
\hline 生麦芽 & Sheng Mai Ya & Hordeum vulgare L. & 15 & $\begin{array}{l}\text { Promotes digestion and invigorate spleen, stop lactation and release } \\
\text { distension }\end{array}$ \\
\hline 益智仁 & Yi Zhi Ren & Fructus Alpinia oxyphylla & 10 & $\begin{array}{l}\text { Tonifies kidney yang, secure essence and reduce urination, warm } \\
\text { spleen yang, improve appetite and reduce salivation }\end{array}$ \\
\hline 香附 & Xiang Fu & Rhizoma Cyperi & 10 & $\begin{array}{l}\text { Unblocks the liver and regulates qi, regulates menstruation and } \\
\text { stops pain }\end{array}$ \\
\hline 远志 & Yuan Zhi & Radix Polygalae & 10 & $\begin{array}{l}\text { Stabilizes the heart and calms the mind, dissolves phlegm and } \\
\text { opens orifices, and reduces abscesses and swelling }\end{array}$ \\
\hline 浙贝母 & Zhe Bei Mu & Bulbus Fritillariae Thunbergii & 10 & $\begin{array}{l}\text { Clears heat and resolves phlegm, disperses masses/abnormal growth } \\
\text { and promotes the healing of carbuncles }\end{array}$ \\
\hline 土获苓 & Tu Fu Ling & Smilax glabra Roxb & 15 & Removes toxicity, excrete dampness and ease joint movement \\
\hline
\end{tabular}

(PRO) and objective blood biomarkers at each time point (Fig. 2). Participants will also be monitored for safety and adverse events (AE).

\section{Assessment of quality of life}

The European Organisation for Research and Treatment of Cancer Quality of Life Questionnaire (EORTC-QLQ 30) is a self-administered questionnaire that measures the health-related QOL. The English and Chinese versions of this questionnaire have been validated in Singaporean cancer patients [32, 33]. It consists of 30 items across five functional domains (physical, role, cognitive, emotional and social), three symptom domains (fatigue, pain and nausea/vomiting), six individual items (dyspnea, insomnia, anorexia, diarrhoea, constipation and financial stability) and a global health status domain. All

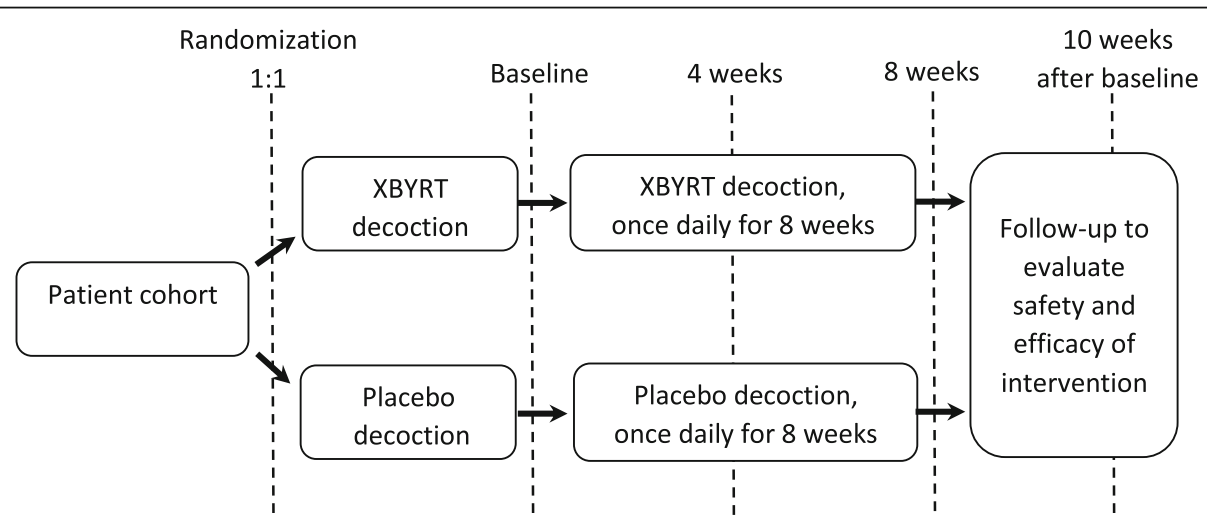

Fig. 1 Flow diagram of the study design and assessment time points 


\begin{tabular}{|c|c|c|c|c|c|c|c|}
\hline & \multicolumn{6}{|c|}{ STUDY PERIOD } & \multirow[b]{2}{*}{ Close-out } \\
\hline & Enrolment & Allocation & \multicolumn{4}{|c|}{ Post-allocation } & \\
\hline TIMEPOINT & \multicolumn{3}{|c|}{ Baseline visit } & \multicolumn{3}{|c|}{ Follow-up visits } & \\
\hline Week & \multicolumn{3}{|c|}{0} & 4 & 8 & 10 & $>10$ \\
\hline \multicolumn{8}{|l|}{ ENROLMENT: } \\
\hline Eligibility screen & $X$ & & & & & & \\
\hline Informed consent & $x$ & & & & & & \\
\hline Allocation & & $\mathrm{X}$ & & & & & \\
\hline \multicolumn{8}{|l|}{ INTERVENTIONS: } \\
\hline \multicolumn{8}{|l|}{ XBYRT intervention } \\
\hline \multicolumn{8}{|l|}{ Placebo } \\
\hline \multicolumn{8}{|l|}{ ASSESSMENTS: } \\
\hline Data Collection Form & $\mathrm{X}$ & & & & & & \\
\hline $\begin{array}{r}\text { TCM Symptom } \\
\text { Scoring }\end{array}$ & & & $\mathrm{X}$ & $\mathrm{X}$ & $X$ & $X$ & \\
\hline EORTC-QLQ-30 & & & $X$ & $\mathrm{X}$ & $\mathrm{X}$ & $X$ & \\
\hline MFSI-SF & & & $X$ & $\mathrm{X}$ & $X$ & $\mathrm{X}$ & \\
\hline FACT-Cog & & & $X$ & $\mathrm{X}$ & $\mathrm{X}$ & $X$ & \\
\hline $\begin{array}{r}\text { Adverse Events } \\
\text { Assessment }\end{array}$ & & & $X$ & $\mathrm{X}$ & $X$ & $X$ & \\
\hline $\begin{array}{r}\text { Blood tests (Full } \\
\text { blood count, liver } \\
\text { and renal function } \\
\text { tests) }\end{array}$ & & & $X$ & $\mathrm{X}$ & $X$ & $X$ & \\
\hline Blood biomarkers & & & $x$ & $x$ & $x$ & $x$ & \\
\hline $\begin{array}{r}\text { Analysis of study } \\
\text { outcomes }\end{array}$ & & & & & & & $\mathrm{X}$ \\
\hline
\end{tabular}

Fig. 2 SPIRIT figure for the enrolment, interventions and assessments of the study

items are linearly transformed into a score ranging from 0 to 100. A higher score represents better functional status and health-related QOL in the functional and global health status domain while a higher score in the symptom domain or individual symptom item is interpreted as a worse extent of symptoms experienced by the patient.

\section{Assessment of fatigue}

The Multidimensional Fatigue Symptom Inventory-Short Form (MFSI-SF) is a multidimensional questionnaire for measuring fatigue in cancer patients. The English and Chinese versions of MFSI-SF have been validated in breast cancer patients in Singapore [34]. This 30-item questionnaire contains five subscales: general fatigue, physical fatigue, emotional fatigue, mental fatigue and vigour. Specific subscale scores are obtained by tabulating the item scores from each subscale. The MFSI-SF subscale scores can be combined to obtain an overall score. A higher score reflects a worse fatigue level.

\section{Assessment of cognition}

The Functional Assessment of Cancer Therapy-Cognitive Function (FACT-Cog) version 3 is used to assess subjective cognitive function. Both the English and Chinese versions of FACT-Cog have been validated in cancer patients in Singapore [35]. This questionnaire has 37 items containing assessments for six domains of cognition (memory, concentration, mental acuity, verbal ability, functional interference 
and multitasking) and two subscales for noticeability and impact on QOL. A global FACT-Cog score is derived from the total of the domain/subscale scores. Higher scores indicate better perceived cognitive abilities and QOL.

\section{TCM symptom scoring}

The TCM symptom scoring form is referenced from the combination of qi and blood deficiency from the National Standards of People Republic of China and details the method of pattern identification [36]. The form contains assessments for 9 major TCM symptoms and 7 possible TCM symptoms (Table 2). These symptoms are scored on an ordinal scale of absent, mild, moderate and severe. The scoring tool will be completed by the assessing TCM physician, and the patient's pulse and tongue appearance will be assessed from the TCM's perspective. Information collected from the questionnaire will be used to screen for inclusion and monitor the response of the participants towards the TCM decoction.

\section{Safety monitoring}

Safety of study participants will be monitored at every time point assessment through blood tests evaluating organ functions such as liver and renal function tests, electrolyte level and full blood count. Participants will be asked to report any adverse effects experienced during the follow-up period which are graded according to the Common Terminology Criteria for Adverse Events (CTCAE) version 5 [37]. There are five grades in ascending order of severity, with grade 1 representing mild symptoms and grade 5 representing death. In order to monitor the adverse events (AEs) or serious adverse events (SAEs), an independent Data and Safety Monitoring Board (DSMB) review will convene for every 20 patients recruited for the trial. Therefore, there will be a total of three DSMB meetings for this trial. Analysis comparing the incidence of AEs based on the intervention group will be conducted at each DSMB review. The DSMB will make recommendations to the study team regarding the appropriate courses of action to address study

Table 2 Major and possible symptoms assessed for TCM symptom scoring

\begin{tabular}{ll}
\hline Major symptoms & Possible symptoms \\
\hline Shortness of breath & Spontaneous sweating \\
General fatigue & Reluctance to talk \\
Weakness & Insomnia \\
Pale or sallow complexion & Limb numbness \\
Dizziness, blurred vision & Scanty menstruation \\
Heart palpitation & Nausea, vomiting \\
Poor appetite & Weakness in defaecation \\
Abdominal distension after food & \\
Loose stools & \\
\hline
\end{tabular}

safety issues which may arise during the trial. Unblinding to the study team is allowed only when the identification of the intervention arm is absolutely necessary for further management of the participant, for example, in the event of a SAE.

\section{Assessment of biomarkers}

Blood samples from participants will be collected for biomarker assessments at all assessment time points.

\section{Mitochondrial DNA content}

The mitochondrial DNA (mtDNA) will be extracted from the buffy coat, and mtDNA content will be evaluated in relation to CRF. To measure mtDNA content, real-time quantitative polymerase chain reaction (PCR) will be performed using the Quantifast SYBR Green Master Mix from Qiagen along with the forward and reverse primers for Mito and $\beta 2$ microglobulin (B2M). The Mito primer pair will amplify the ZFP28 zinc finger protein gene in $\mathrm{mtDNA}$, while the $\mathrm{B} 2 \mathrm{M}$ primer pair targets the beta-2-microglobulin gene in nuclear DNA (nDNA). The analysis will be performed in triplicates on 96-well plates, and target sequences will be amplified in a Biorad CFX96 Touch cycler. The average threshold cycle $(\mathrm{Ct})$ values of nuclear DNA and mtDNA from each triplicate series are obtained, and the $\Delta \mathrm{Ct}$ method is applied $(\Delta \mathrm{Ct}=\mathrm{CtnDNA}-\mathrm{CtmtDNA})$. The relative mtDNA copy number is derived from $2 \times 2^{\Delta \mathrm{Ct}}$, taking into account that nDNA is diploid.

\section{Inflammatory cytokines}

Plasma levels of inflammatory marker $\mathrm{C}$-reactive protein (CRP) and cytokines TNF- $\alpha$, IL-1 $\beta$, IL- 6 and IL- 8 will be measured. The choice of cytokines is based on the findings of a systematic review which suggest that the development of CRF is influenced by immune dysregulation of which the aforementioned cytokines have been shown to contribute to worsening or persistent fatigue [8]. The cytokines of interest will be quantified using the highly sensitive multiplex immunoassay (Luminex ${ }^{\circ}$ ).

\section{Oxidative stress markers}

Plasma levels of malondialdehyde (MDA), superoxide dismutase (SOD) and glutathione peroxidase (GSH-Px) will be measured using commercially available assay kits.

\section{Outcomes}

\section{Primary outcome}

The primary endpoint is the difference in global health status (GHS) score between the XBYRT interventional and placebo arms from baseline to 8 weeks after baseline. The GHS is a two-item domain from the EORTCQLQ 30. 


\section{Secondary outcomes}

Secondary outcomes are the changes in MFSI-SF and FACT-Cog total scores, including the subscale or domain scores between the XBYRT and placebo arms from baseline to 4, 8 and 10 weeks after baseline. Changes in the functional and symptom domain scoring of the EORTC QLQ-C30 from baseline will also be evaluated. Safety outcomes will be reported as the incidence of AEs or SAEs in study participants.

\section{Biomarkers}

Changes in circulating levels of inflammatory markers CRP, TNF- $\alpha$, IL-1 $\beta$, IL- 6 and IL- 8 from baseline to 4,8 and 10 weeks after baseline will be compared between the XBYRT and placebo arms. Circulating oxidative stress markers, malondialdehyde (MDA), superoxide dismutase (SOD) and glutathione peroxidase (GPx), as well as mtDNA levels, will also be compared between the XBYRT and placebo arms for the aforementioned time points.

\section{Data management}

The research data will be entered into a passwordprotected electronic database, and the entered data will be checked with the source documents to ensure data accuracy, by an independent study team member who is not in charge of data entry. The hard copy of study records or electronic study database will be kept in controlled access locations under lock and key. Investigators involved in this study will have access to the final dataset.

\section{Statistical analysis}

The difference in GHS scores at 8 weeks after baseline between XBYRT intervention and placebo will be assessed using independent $t$ test. To assess for the longitudinal effect of score changes over time, linear mixed models will be used to compare the outcomes between XBYRT intervention and placebo at the respective time points, adjusting for baseline values and clinically relevant factors. Individualspecific effects will be treated as random effects. Biomarker levels will also be evaluated using $t$ tests and linear mixed models. The safety outcomes will be reported in proportions of patients experiencing AEs (\% in terms of incidence) using the CTCAE v5.0 Criteria. Chi-square test will be used to evaluate the difference in severity (by grades) between the XBYRT intervention and placebo groups. An intentionto-treat (ITT) analysis approach will be adopted, and the data from all participants who are randomized and completed the trial without any major violation of the protocol will be analysed. Missing data will be treated as missing completely at random.

\section{Study dissemination}

The findings from this trial will be disseminated through conference presentations and publications in peerreviewed journals.

\section{Patient and public involvement}

Patients or the public were not involved in the design, conduct or reporting of the research.

\section{Discussion}

Currently, CRF is often under-reported and under-treated as there is a limited understanding of effective management strategies for the symptoms [38]. The current nonpharmacological recommendations for managing CRF include exercise, cognitive behavioural therapy and patient education [39, 40]. However, pharmacological therapies are still investigational and have limited efficacy [39, 40]. Hence, there is a need to gain a clearer understanding of CRF aetiology and identify therapies that could effectively manage CRF in cancer survivors.

TCM presents an attractive therapeutic option for the management of CRF symptoms as the concept of using TCM decoctions to replenish the qi or the body vital energy and improve sleep disturbances has been recorded in classical traditional Chinese medical texts [41]. The biological effects of TCM have been investigated in a number of in vitro and in vivo studies, and among the proposed mechanisms for the positive effects of herbal TCM on fatigue are the anti-inflammatory, immuno-modulatory and antioxidant abilities of the herbal components [41-43]. For example, supplementation with Radix Astragali extract enhanced endurance and reduced the levels of reactive oxygen species and cytokines in mice and rats with exerciseinduced fatigue [42, 43]. One of the main TCM uses of Radix Paeoniae Alba is to treat blood deficiency, and an in vivo investigation has demonstrated that Radix Paeoniae Alba extract increased haemoglobin, haematocrit and serum erythropoietin in anaemia-induced rats [44]. Phytochemicals extracted from Radix Codonopsis Pilosulae, Rhizoma Atractylodis Macrocephalae, Poria and Fructus Lycii also exerted anti-inflammatory, immuno-modulatory and antioxidative activities in vitro and in vivo [45-48]. Therefore, the analysis of the cytokines, antioxidant markers and mtDNA levels of participants in the XBYRT intervention and placebo arms in this trial will determine the effects of the XBYRT decoction on the inflammatory and oxidative stress status of patients with CRF. Cytokines and mtDNA are also potential predictive biomarkers for CRF $[5,7,14]$. These markers are useful as they will provide an objective observation on the patients' response to the TCM concoction.

Although in vitro and in vivo studies have shown evidence supporting the purported effects of these herbal medicines, there is still a dearth of clinical evidence due 
to the limited number of clinical trials investigating the use of herbal TCM in treating CRF. In a single-arm study, the use of Ren Shen Yangrong Tang (RSYRT) was investigated in 33 cancer survivors who have reported moderate to severe level of fatigue [20]. RSYRT, which contains 12 herbal components, was formulated to correct qi deficiency, and the formulation consists of some similar herbal ingredients to the XBYRT used in this protocol, such as the Radix Codonopsis, Rhizoma Atractylodis Macrocephalae, Radix Paeoniae Alba, Poria Cocos and Radix Polygalae. A significant decrease in fatigue severity was seen in patients after 6 weeks of treatment with RSYRT [20]. In a randomized controlled trial, 40 patients with CRF were randomized to receive either an intervention, Bu-Zhong-Yi-Qi-Tang (BZYQT) or no intervention. BZYQT contains 10 herbs with Radix Astragali, Atractylodis lanceae rhizome and Radix Ginseng as the main components [21]. Patients who received BZYQT for 2 weeks reported significant improvements in fatigue levels and QOL [21]. Although these studies reported positive outcomes, they were not adequately controlled to eliminate possible bias or placebo effects.

Therefore, the outcome from this randomized, doubleblind, placebo-controlled clinical trial will contribute valuable clinical evidence on the efficacy and safety of a TCM decoction for managing CRF symptoms and QOL in a cohort of cancer survivors. Results obtained from this study will also generate important data for the design of a larger phase III clinical trial.

\section{Conclusion}

In summary, this HERBAL study evaluates the efficacy of a modified TCM decoction, the XBYRT, which is specifically formulated for addressing qi and blood deficiency to manage CRF symptoms in cancer survivors. The results from this clinical trial can provide both clinical and biochemical basis and guidance for future largescale randomized controlled trials and facilitate a better understanding of possible biological mechanisms contributing to the effects of TCM.

\section{Trial status}

At the time of submission, recruitment is still ongoing. The recruitment and follow-up period for this trial will be from October 2019 to December 2021.

At manuscript submission, the study protocol used is the version 10 (15 October 2020), approved on 23 October 2020.

\section{Supplementary Information}

Supplementary information accompanies this paper at https://doi.org/10. 1186/s13063-020-04810-4.

Additional file 1. SPIRIT 2013 Checklist: Recommended items to address in a clinical trial protocol and related documents.

\section{Abbreviations}

AE: Adverse event; B2M: $\beta 2$ microglobulin; CAM: Complementary and alternative medicine; CRF: Cancer-related fatigue; CRP: C-reactive protein; CTCAE: Common Terminology Criteria for Adverse Events; DSMB: Data and Safety Monitoring Board; EORTC QLQ-C30: European Organization for Research and Treatment of Cancer Quality of Life Questionnaire; FACTCog: Functional Assessment of Cancer Therapy-Cognitive Function; GHS: Global health status; GSH-Px: Glutathione peroxidase; IL: Interleukin; MDA: Malondialdehyde; MFSI-SF: Multidimensional Fatigue Symptom Inventory-Short Form; mtDNA: Mitochondrial DNA; NCCS: National Cancer Centre Singapore; PCR: Polymerase chain reaction; PRO: Patient-reported outcomes; QOL: Quality of life; SAE: Serious adverse event; SD: Standard deviation; SOD: Superoxide dismutase; TCM: Traditional Chinese medicine; XBYRT: Xiang Bei Yang Rong Tang

\section{Acknowledgements \\ Not applicable}

\section{Authors' contributions}

1. NYY: drafting of the manuscript, protocol design and amendments.

2. WSL: manuscript editing and approval and protocol amendments.

3. HFZ: manuscript editing and approval and protocol design.

4. QMT: manuscript editing and approval and protocol design.

5. TKT: manuscript editing and approval and protocol design.

6. LYPQ: manuscript editing and approval and, protocol design.

7. CJT: manuscript editing and approval and protocol design.

8. YLT: manuscript editing and approval and protocol design.

9. CCN: manuscript editing and approval and protocol design.

10. SKA: manuscript editing and approval.

11. VKMT: manuscript editing and approval.

12. HKH: manuscript editing and approval.

13. LC: manuscript editing and approval.

14. KWJL: manuscript editing and approval and protocol design.

15. TJYT: manuscript editing and approval and protocol design

16. AC: manuscript editing and approval and protocol design.

The authors read and approved the final manuscript.

\section{Funding}

This trial is funded by the Singapore Ministry of Health Traditional Chinese Medicine Research Grant (TCMRG-3-NUS-01). The funder was not involved in the design of the protocol and the decision to submit the protocol for publication.

Availability of data and materials

This is an investigator-initiated trial; therefore, the investigators will have access to the trial dataset.

\section{Ethics approval and consent to participate}

This study protocol has received ethical approval from the Singhealth Centralised Institutional Review Board (2019/2135). Written informed consent will be obtained from all study participants.

\section{Consent for publication}

No details, images or videos relating to an individual person will be published.

\section{Competing interests}

The authors declare that they have no competing interests.

\section{Author details}

'Department of Pharmacy, National University of Singapore, Singapore, Singapore. ${ }^{2}$ Department of Pharmacy, National Cancer Centre Singapore, Singapore, Singapore. ${ }^{3}$ Singapore Thong Chai Medical Institution, Singapore, Singapore. ${ }^{4}$ Division of Surgery \& Surgical Oncology, National Cancer Centre Singapore, Singapore, Singapore. ${ }^{5}$ Division of Medical Oncology, National Cancer Centre Singapore, Singapore, Singapore. ${ }^{6}$ Department of Clinical Pharmacy Practice, University of California, Irvine, USA. 
Received: 14 August 2020 Accepted: 16 October 2020 Published online: 04 November 2020

\section{References}

1. Bray F, Ferlay J, Soerjomataram I, Siegel RL, Torre LA, Jemal A. Global cancer statistics 2018: GLOBOCAN estimates of incidence and mortality worldwide for 36 cancers in 185 countries. CA Cancer J Clin. 2018;68(6):394-424.

2. Curt GA, Breitbart W, Cella D, Groopman JE, Horning SJ, Itri LM, Johnson DH, Miaskowski C, Scherr SL, Portenoy RK, et al. Impact of cancer-related fatigue on the lives of patients: new findings from the Fatigue Coalition. Oncologist. 2000;5(5):353-60.

3. Garabeli Cavalli Kluthcovsky AC, Urbanetz AA, de Carvalho DS, Pereira Maluf EM, Schlickmann Sylvestre GC, Bonatto Hatschbach SB. Fatique after treatment in breast cancer survivors: prevalence, determinants and impact on health-related quality of life. Support Care Cancer. 2012;20(8):1901-9.

4. Wang XS, Zhao F, Fisch MJ, O'Mara AM, Cella D, Mendoza TR, Cleeland CS. Prevalence and characteristics of moderate to severe fatigue: a multicenter study in cancer patients and survivors. Cancer. 2014;120(3):425-32.

5. Pertl MM, Hevey D, Boyle NT, Hughes MM, Collier S, O'Dwyer AM, Harkin A, Kennedy MJ, Connor TJ. C-reactive protein predicts fatigue independently of depression in breast cancer patients prior to chemotherapy. Brain Behav Immun. 2013:34:108-19.

6. Orre IJ, Reinertsen KV, Aukrust P, Dahl AA, Fossa SD, Ueland T, Murison R. Higher levels of fatigue are associated with higher CRP levels in disease-free breast cancer survivors. J Psychosom Res. 2011;71(3):136-41.

7. Liu L, Mills PJ, Rissling M, Fiorentino L, Natarajan L, Dimsdale JE, Sadler GR, Parker BA, Ancoli-lsrael $\mathrm{S}$. Fatigue and sleep quality are associated with changes in inflammatory markers in breast cancer patients undergoing chemotherapy. Brain Behav Immun. 2012;26(5):706-13.

8. Eyob T, Ng T, Chan R, Chan A. Impact of chemotherapy on cancer-related fatigue and cytokines in 1312 patients: a systematic review of quantitative studies. Curr Opin Support Palliat care. 2016;10(2):165-79.

9. Gilliam LA, St Clair DK. Chemotherapy-induced weakness and fatigue in skeletal muscle: the role of oxidative stress. Antioxid Redox Signal. 2011; 15(9):2543-63.

10. Lacourt TE, Vichaya EG, Chiu GS, Dantzer R, Heijnen CJ. The high costs of low-grade inflammation: persistent fatigue as a consequence of reduced cellular-energy availability and non-adaptive energy expenditure. Front Behav Neurosci. 2018;12(78).

11. Vichaya EG, Chiu GS, Krukowski K, Lacourt TE, Kavelaars A, Dantzer R, Heijnen CJ, Walker AK. Mechanisms of chemotherapy-induced behavioral toxicities. Front Neurosci. 2015;9(131).

12. Booth NE, Myhill S, McLaren-Howard J. Mitochondrial dysfunction and the pathophysiology of myalgic encephalomyelitis/chronic fatigue syndrome (ME/CFS). Int J Clin Exp Med. 2012;5(3):208-20.

13. Filler K, Lyon D, McCain N, Bennett J, Fernández-Martínez JL, deAndrésGaliana EJ, Elswick RK, Lukkahatai N, Saligan L. Relationship of mitochondrial enzymes to fatigue intensity in men with prostate cancer receiving external beam radiation therapy. Biol Res Nurs. 2016;18(3):274-80.

14. Chae JW, Chua PS, Ng T, Yeo AHL, Shwe M, Gan YX, Dorajoo S, Foo KM, Loh $\mathrm{KW}$, Koo SL, et al. Association of mitochondrial DNA content in peripheral blood with cancer-related fatigue and chemotherapy-related cognitive impairment in early-stage breast cancer patients: a prospective cohort study. Breast Cancer Res Treat. 2018;168(3):713-21.

15. Shih V, Chiang JY, Chan A. Complementary and alternative medicine (CAM) usage in Singaporean adult cancer patients. Ann Oncol. 2009;20(4):752-7.

16. Kiang $L C$, Chiang J, Shih V, Chan A. The prevalence of complementary and alternative medicine (CAM) usage in Singaporean breast cancer patients. Asia Pacific J Onc Hematol. 2009:1(3):11-6.

17. Carmady B, Smith CA. Use of Chinese medicine by cancer patients: a review of surveys. Chin Med. 2011;6:22

18. Hsu CH, Lee CJ, Chien TJ, Lin CP, Chen CH, Yuen MJ, Lai YL. The relationship between qi deficiency, cancer-related fatigue and quality of life in cancer patients. J Tradit Complement Med. 2012;2(2):129-35.

19. Yeh M-H, Chao C-H, Koo M, Chen C-Y, Yeh C-C, Li T-M. Association of traditional Chinese medicine body constitution and moderate-to-severe cancer-related fatigue in cancer patients. Complement Ther Med. 2019;43: 44-8.

20. Xu Y, Chen Y, Li P, Wang XS. Ren Shen Yangrong Tang for fatigue in cancer survivors: a phase I/II open-label study. J Altern Complement Med (New York, NY). 2015;21(5):281-7.
21. Jeong JS, Ryu BH, Kim JS, Park JW, Choi WC, Yoon SW. Bojungikki-tang for cancer-related fatique: a pilot randomized clinical trial. Integr Cancer Ther. 2010;9(4):331-8.

22. Zhao H, Zhang Q, Zhao L, Huang X, Wang J, Kang X. Spore powder of Ganoderma lucidum improves cancer-related fatigue in breast cancer patients undergoing endocrine therapy: a pilot clinical trial. Evid Based Complement Altern Med. 2012;2012:809614.

23. Barton DL, Liu H, Dakhil SR, Linquist B, Sloan JA, Nichols CR, McGinn TW, Stella PJ, Seeger GR, Sood A, et al. Wisconsin ginseng (Panax quinquefolius) to improve cancer-related fatigue: a randomized, double-blind trial, N07C2. J Natl Cancer Inst. 2013;105(16):1230-8.

24. Wu Q. Yi Zong Jin Jian, vol. 64. Beijing: People's Medical Publishing House; 2006.

25. Dai L, Cheng CW, Tian R, Zhong LL, Li YP, Lyu AP, Chan AW, Shang HC, Bian ZX. Standard Protocol Items for Clinical Trials with Traditional Chinese Medicine 2018: recommendations, explanation and elaboration (SPIRIT-TCM extension 2018). Chin J Integr Med. 2019;25(1):71-9.

26. Bower JE, Bak K, Berger A, Breitbart W, Escalante CP, Ganz PA, Schnipper HH, Lacchetti C, Ligibel JA, Lyman GH. Screening, assessment, and management of fatigue in adult survivors of cancer: an American Society of Clinical Oncology clinical practice guideline adaptation. J Clin Oncol. 2014;32(17):1840.

27. Pharmacopoeia Commission of the Ministry of Health of the People's Republic of China: Pharmacopoeia of the People's Republic of China. China; 2015

28. Gao XM. General eleventh five-year national planning textbook for higher education: Chinese Materia Medica. Beijing: China Press of Traditional Chinese Medicine; 2009

29. Wang $Q$, Wang YR, Jia QY, Liu L, Xu CQ, Wang XY, Yao M, Cui XJ, Shi Q, Wang $Y$ J, et al. The efficacy of the traditional Chinese medicine Juanbi pill combined with methotrexate in active rheumatoid arthritis: study protocol for a randomized controlled trial. Trials. 2018;19(1):188.

30. Liu Q-Q, Zhang J, Guo R-J, Xie Y-Z, Fu Q-N, He T, Zhu X-Q, Du J, Yang J, Wang J-L, et al. Efficacy and safety of the Chaihuguizhiganjiang-suanzaoren granule on primary insomnia: study protocol for a randomised controlled trial. BMJ Open. 2016;6(2):e008459.

31. Teare MD, Dimairo M, Shephard N, Hayman A, Whitehead A, Walters SJ. Sample size requirements to estimate key design parameters from external pilot randomised controlled trials: a simulation study. Trials. 2014;15:264.

32. Cheung YB, Thumboo J, Goh C, Khoo KS, Che W, Wee J. The equivalence and difference between the English and Chinese versions of two major, cancer-specific, health-related quality-of-life questionnaires. Cancer. 2004; 101(12):2874-80.

33. Luo N, Fones CS, Lim SE, Xie F, Thumboo J, Li SC. The European Organization for Research and Treatment of Cancer Quality of Life Questionnaire (EORTC QLQ-c30): validation of English version in Singapore. Qual Life Res. 2005;14(4):1181-6.

34. Chan A, Lew C, Wang XJ, Ng T, Chae JW, Yeo HL, Shwe M, Gan YX. Psychometric properties and measurement equivalence of the Multidimensional Fatigue Syndrome Inventory-Short Form (MFSI-SF) amongst breast cancer and lymphoma patients in Singapore. Health Qual Life Outcomes. 2018;16(1):20.

35. Cheung YT, Lim SR, Shwe M, Tan YP, Chan A. Psychometric properties and measurement equivalence of the English and Chinese versions of the functional assessment of cancer therapy-cognitive in Asian patients with breast cancer. Value Health. 2013;16(6):1001-13.

36. Lin HS. Breast cancer. In: Chinese practice guidelines of Chinese medicine in oncology. China: People's Medica Publishing House; 2016. p. 129-38.

37. National Institutes of Health/National Cancer Institute: Common terminology criteria for adverse events (CTCAE) version 5.0. 2017.

38. James S, Wright $P$, Scarlett $C$, Young T, Jamal H, Verma R. Cancer-related fatigue: results from patient experience surveys undertaken in a UK regional cancer centre. Support Care Cancer. 2015;23(7):2089-95.

39. Mustian KM, Alfano CM, Heckler C, Kleckner AS, Kleckner IR, Leach CR, Mohr D, Palesh OG, Peppone $L$, Piper BF, et al. Comparison of pharmaceutical, psychological, and exercise treatments for cancer-related fatigue: a metaanalysis. JAMA Oncol. 2017;3(7):961-8.

40. NCCN Guidelines: National Comprehensive Cancer Network (NCCN) Clinical Practice Guidelines in Oncology, cancer-related fatique version 2. 2018.

41. Chen R, Moriya J, Yamakawa J-I, Takahashi T, Kanda T. Traditional Chinese medicine for chronic fatique syndrome. Evid Based Complement Altern Med. 2010;7(1):3-10. 
42. Kuo YH, Tsai WJ, Loke SH, Wu TS, Chiou WF. Astragalus membranaceus flavonoids (AMF) ameliorate chronic fatigue syndrome induced by food intake restriction plus forced swimming. J Ethnopharmacol. 2009;122(1):28-34.

43. Lee JS, Kim HG, Han JM, Kim YA, Son CG. Anti-fatique effect of Myelophil in a chronic forced exercise mouse model. Eur J Pharmacol. 2015;764:100-8.

44. Lee HW, Kim H, Ryuk JA, Kil K-J, Ko BS. Hemopoietic effect of extracts from constituent herbal medicines of Samul-tang on phenylhydrazine-induced hemolytic anemia in rats. Int J Clin Exp Pathol. 2014;7(9):6179-85.

45. Deng X, Fu Y, Luo S, Luo X, Wang Q, Hu M, Ma F, Ma CW, Zhou L. Polysaccharide from Radix Codonopsis has beneficial effects on the maintenance of T-cell balance in mice. Biomed Pharmacother. 2019:112:108682.

46. Li X, Lin J, Han W, Mai W, Wang L, Li Q, Lin M, Bai M, Zhang L, Chen D. Antioxidant ability and mechanism of rhizoma Atractylodes macrocephala. Molecules (Basel, Switzerland). 2012;17(11):13457-72.

47. RuiDian K, ShunFa L, Yi C, ChuRong J, OiaGuang S. Analysis of chemical composition of polysaccharides from Poria cocos Wolf and its anti-tumor activity by NMR spectroscopy. Carbohydr Polym. 2010;80(1):31-4.

48. Cui B, Liu S, Lin X, Wang J, Li S, Wang Q, Li S. Effects of Lycium barbarum aqueous and ethanol extracts on high-fat-diet induced oxidative stress in rat liver tissue. Molecules (Basel, Switzerland). 2011;16(11):9116-28.

\section{Publisher's Note}

Springer Nature remains neutral with regard to jurisdictional claims in published maps and institutional affiliations.

Ready to submit your research? Choose BMC and benefit from:

- fast, convenient online submission

- thorough peer review by experienced researchers in your field

- rapid publication on acceptance

- support for research data, including large and complex data types

- gold Open Access which fosters wider collaboration and increased citations

- maximum visibility for your research: over $100 \mathrm{M}$ website views per year

At $\mathrm{BMC}$, research is always in progress.

Learn more biomedcentral.com/submissions 